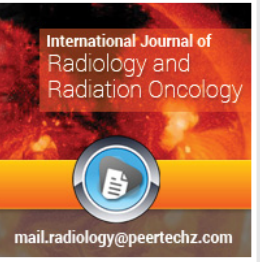

Clinical Group

\title{
International Journal of Radiology and Radiation Oncology
}

DOI http://dx.doi.org/10.17352/ijrro.000018

\section{Jialin Yuan and Jingshan Gong*}

Department of Radiology, Shenzhen People's Hospital, Second Clinical Medical College, Jinan University, China

Dates: Received: 13 December, 2016; Accepted: 29 December, 2016; Published: 30 December, 2016

*Corresponding author: Jingshan Gong, Department of Radiology, Shenzhen People's Hospital, Second Clinical Medical College, Jinan University, Dongmenbeilu 1017, Shenzhen, 518020, China, Tel: +86-13631635669; +86-755-22942883; Fax: +86-75525533497; E-mail: jshgong@sina.com

Keywords: Spleen; Sclerosing angiomatoid nodular transformation; Computed tomography; Magnetic resonance imaging; Diagnosis

https://www.peertechz.com

\section{Case Report}

\section{Sclerosing Angiomatoid Nodular} Transformation of the Spleen (SANT): CT and MR Imaging Features of Five Cases with Pathological Correlation

\section{Abstract}

Objective: To describe imaging features of sclerosing angiomatoid nodular transformation (SANT) with pathologic correlation.

Methods: Imaging findings of five cases of SANT were retrospectively analyzed and compared with pathological manifestations.

Results: All the five cases of SANT were solitary and round masses with maximum diameter ranged from $3 \mathrm{~cm}$ to $9 \mathrm{~cm}$. In ultrasound, all the lesions were well circumscribed with one of hypoecho and four of hyperecho with posterior enhancement. Two masses were of hypoattenuation and one was of isoattenuation at pre-contrast CT scanning. At MRI, the two masses appeared isointensity on T1 weighted images, and hypointense nodules with diffuse signal void spots on T2 weighted images. After intravenous contrast materials, all the five masses showed peripheral circle enhancement at the hepatic arterial phase. The enhanced circle persisted and radial enhanced lines occured in three masses and progressive filling in 2 masses at the portal vein phase. At the delay phase, progressive enhancement resulted in two isodensity masses and one hypoattenuation mass with radial enhanced lines at CT, and one obvious hyperintensity mass with central hypointensty scar and one spoke -like appearance enhancement at MR images. The progressive enhancement and hypointensity at T2 weighted MR images were coresponded to abundent fibrous stroma at pathological examinations, while the signal void might result from hemosiderin in the angiomatoid nodules.

Conclusions: CT and MRI can demonstrate some characteristic findings of SANT, which can facilitate radiologists to make correct diagnosis.

\section{Introduction}

Sclerosing angiomatoid nodular transformation (SANT) is a rare benign vascular lesion of the spleen with extensive sclerosis and unknown etiology, characterized microscopically by multiple angiomatoid nodules embedded within fibrosclerotic stroma [1]. The imaging manifestations of SANT have been limited in the radiologic literature. Herein, we present a series of five patients with incidentally found SANT. Imaging features were described with pathological correlation.

\section{Materials and Methods}

The Ethnic Commission of Shenzhen People's Hospital approved this study and written informed consent was obtained before CT or MRI examination. Imaging findings of five patients with pathological proven SANT were retrospectively analyzed.
The five patients included 3 men and 2 women with age ranged from 30 to 56 years old. All the splenic lesions were incidentally detected by ultrasound. Their clinical history and laboratory examinations were unremarkable. Contrast-enhanced CT was performed in 3 patients and contrast-enhanced MRI in 2 patients.

\section{Results}

Solitary well-circumscribed masses were detected in all the five patients by ultrasound. One mass was of hypoecho, while the remaining masses were of hyperecho with posterior enhancement (Figure $1 \mathrm{~A}$ ). The maximum diameter ranged from $3.0 \mathrm{~cm}$ to $9.0 \mathrm{~cm}$. At CT scanning, two masses appeared as hypodense (Figure $1 \mathrm{~B}$ ) and one was isodense (Figure $2 \mathrm{~A}$ ) to the normal spleenic parenchyma. In the two patients who received MR imaging, masses were isointensity at T1 weighted 
images (Figure 3A) and hypointensity with scattered sign void spots at T2 weighted images (Figure $3 \mathrm{~B}$ ). After intravenous injection of the contrast materials, all the five masses presented circle enhancement at arterial phase (Figure $3 \mathrm{C}$ and Figure $4 \mathrm{~A}$ ), while peripheral enhanced node was noted in one mass (Figure $2 \mathrm{~B}$ ). The circle enhancement persisted and radical line enhancement were observed at portal vein phase in 3 cases (Figure $1 \mathrm{C}$ ) and progressive filling in was seen in two cases (Figure $2 \mathrm{C}$ ). At delay phase of MR imaging, one lesion

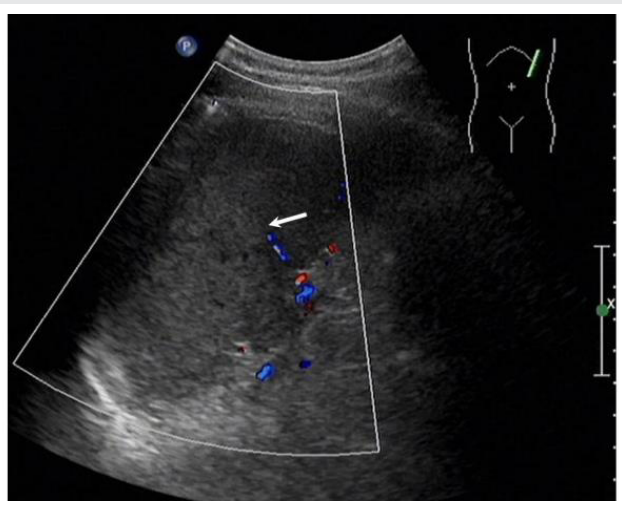

Figure 1a: 50-year-old man with SANT. Ultrasound shows a well circumscribed hyperechoic mass with posterior enhancement (arrow) in the center of the spleen.

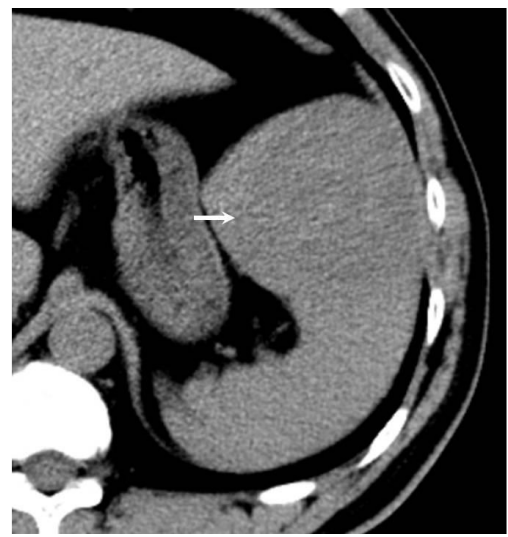

Figure 1b: 50-year-old man with SANT. Non enhanced CT image demonstrates a hypo-attenuation mass with ill-defined margin (arrow) in the front of the spleen.

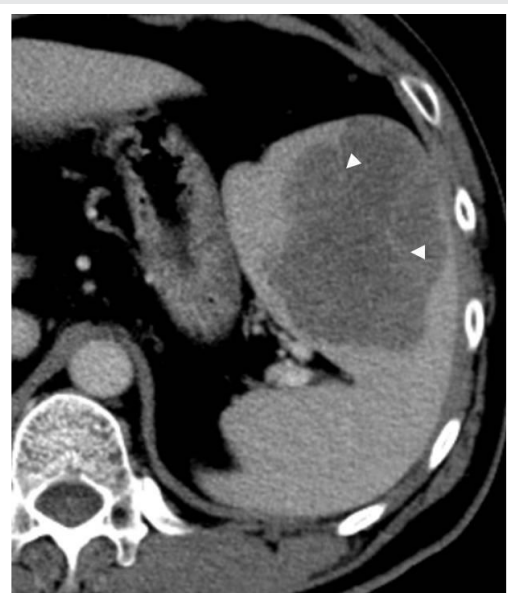

Figure 1c: 50-year-old man with SANT. Contrast-enhanced CT image at the delay phase showing the mass was of hypoattenuation with radial enhanced lines (arrowheads)

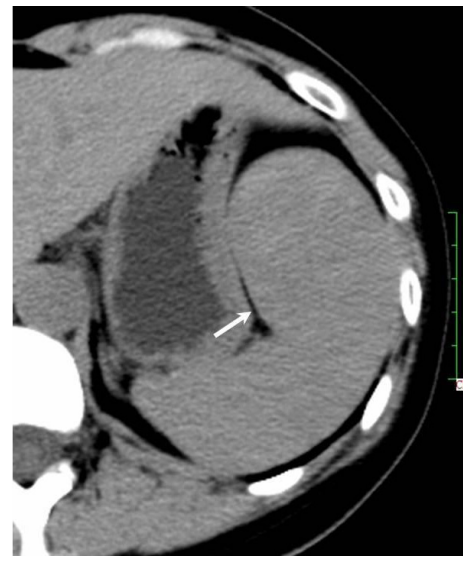

Figure 2a: 40-year-old woman with SANT. Non enhanced axial CT image shows no obvious lession was seen except hypertrophy of the anterior portion of the spleen (arrow)

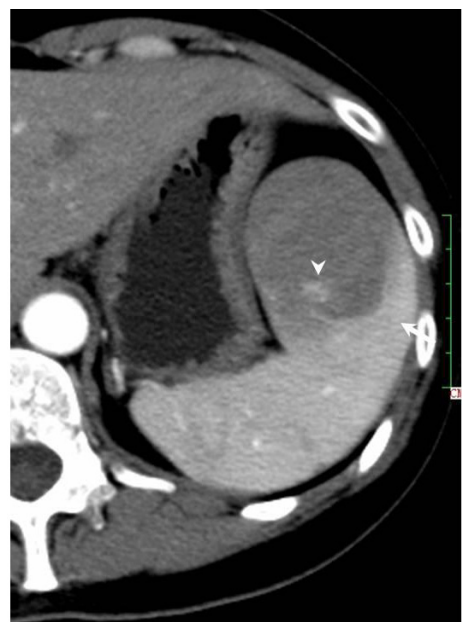

Figure 2b: 40-year-old woman with SANT. Contrast-enhanced transversal CT image at the hepatic arterial phase reveals a well-demarcated hypoattenuation mass with perpheral enhanced circle (arrow) and node (arrowhead).

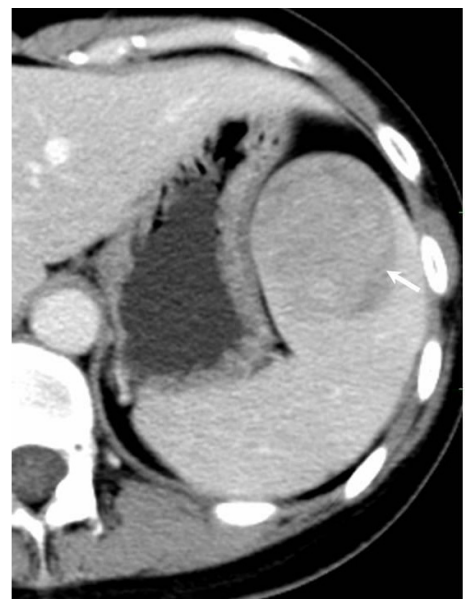

Figure 2c: 40-year-old woman with SANT. Contrast-enhanced transversal CT image at the portal vein phase demonstrates the enhancement progressively filling in (arrow).

showed obvious enhancement with stellate low signal scar in the center comparing with surrounding splenic parenchyma (Figure 3D), while the other demonstrated central and circle 
enhancement to form a spoke-like appearance (Figure 4B). At delay enhanced CT scanning, two masses were isoattenuation to the surrounding splenic parenchyma (Figure 2D) and one lesion kept as hypoattenuation but with radial enhanced lines and enhanced circle to form a spoke-like appearance.

At pathological examination, all the masses were well demarcated on gross cut surface. A central stellate scar with peripheral interspersed dark brown nodules and the whitish intervening fibrous bands was noted to form spoke-like appearance (Figure $3 \mathrm{E}$ ), which was well correlated with CT and MR imaging findings at enhancement. Microscopically, these angiomatoid nodules were surrounded by dense concentric collagen fibrous stroma and dispersed stromal cells with oval-to-spindle nuclei and scanty cytoplasm (Figure $3 \mathrm{~F}$ ). The abunden fibrous stroma may result in hypointensity at T2 weighted MR images. Hemosiderin was noted in the angiomatoid nodules (Figure $3 \mathrm{G}$ ), which might contributed to signal void at T2 weighted MR images.

\section{Discussion}

The term SANT first appeared in the literature in a 2004 paper by Martel et al. [1], which examined a series of 25 cases. This relatively uncommon splenic lesion had however been

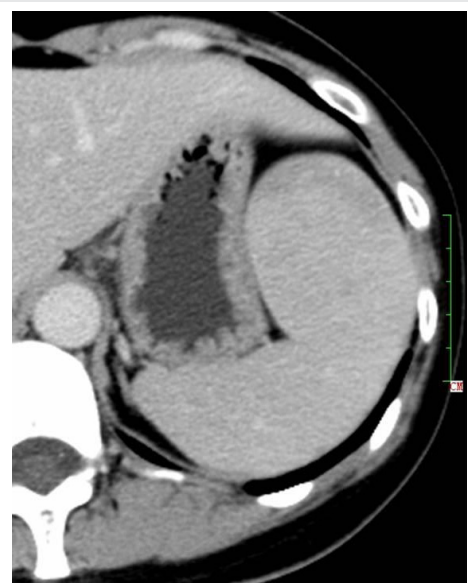

Figure 2d: 40-year-old woman with SANT. Contrast-enhanced transversal CT image at the delay phase shows the mass is almost isoattenuation to surrounding parenchyma of the spleen.

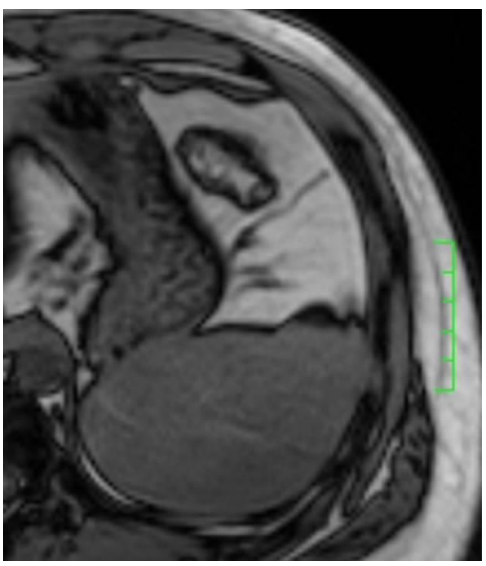

Figure 3a: 30-year-old man with SANT. T1 weighted image shows no obvious lesion at the spleen.

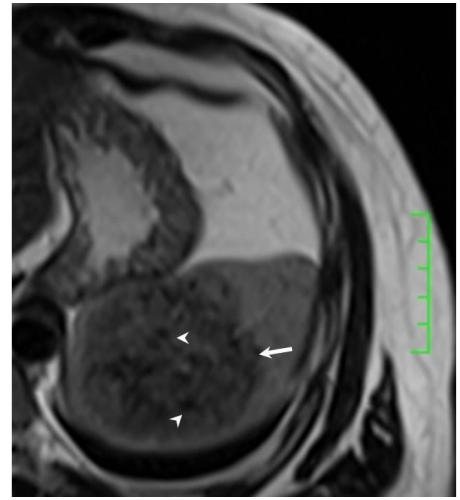

Figure 3b: 30-year-old man with SANT. T2 weighted image reveals a welldemarcated hypointensity mass (arrow) in the spleen. Multiple signal void spots (arrowheads) distributed scanteredly in the mass.

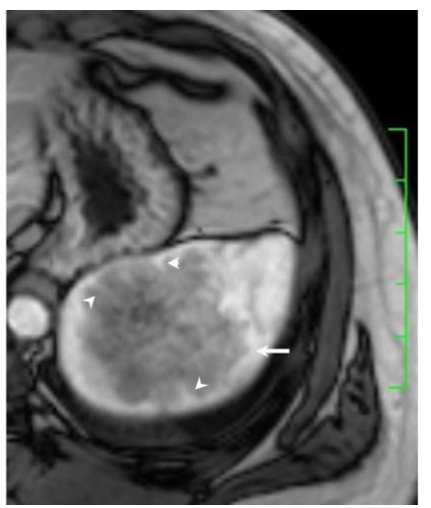

Figure 3c: 30-year-old man with SANT. Contrast enhanced T1 weighted image at the arteral phase shows circle enhancement (arrow) and radical enhanced lines (arrowheads)

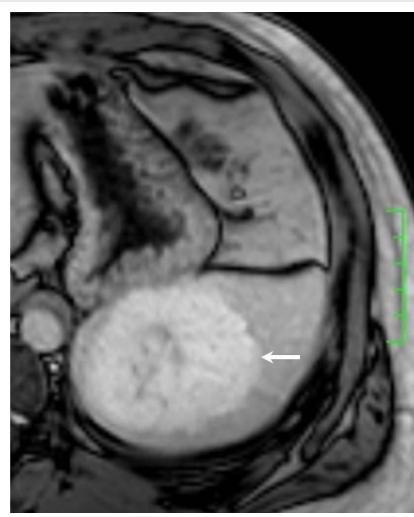

Figure 3d: 30-year-old man with SANT. Contrast enhanced T1 weighted image at the delay phase shows obvious delay enhancement of the mass (arrow).

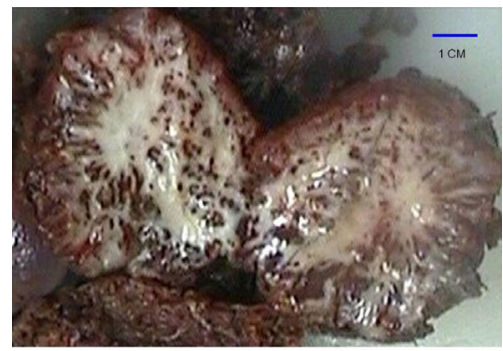

Figure 3e: 30-year-old man with SANT. Photograph of gross pathologic specimen shows solitary well-circumscribed mass with multiple brown nodules separated by pale fibrous stroma. 


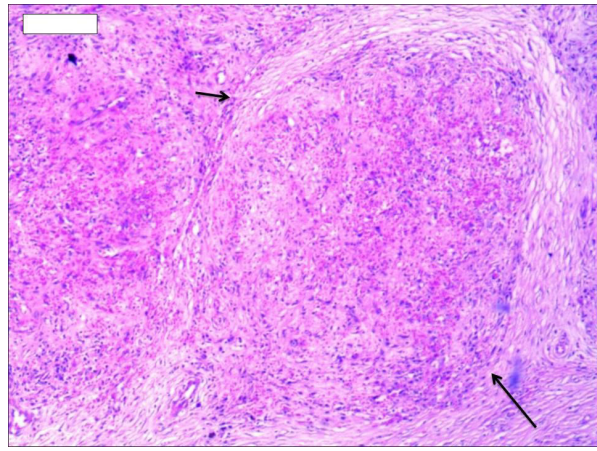

Figure 3f: 30-year-old man with SANT. Photomicrograph ( $\times 100$, hematoxylin and eosin) the central portion of one of the nodules shows proliferation of irregular vascular spaces (long arrow) surrounded by fibrous stroma( short arrow).

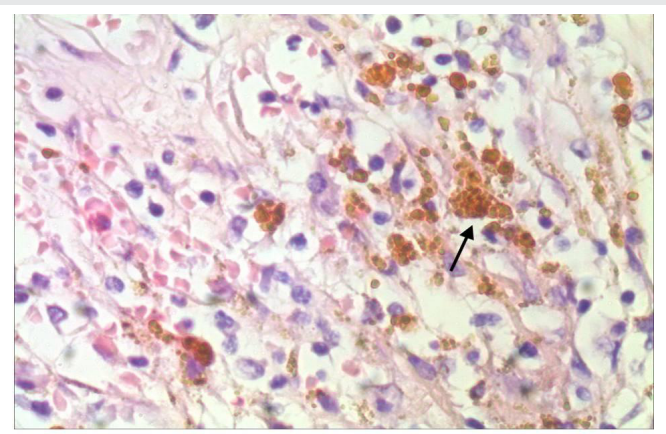

Figure 3g: 30-year-old man with SANT. High-power photomicrograph $(\times 400$ hematoxylin and eosin) shows hemosiderin (arrow).

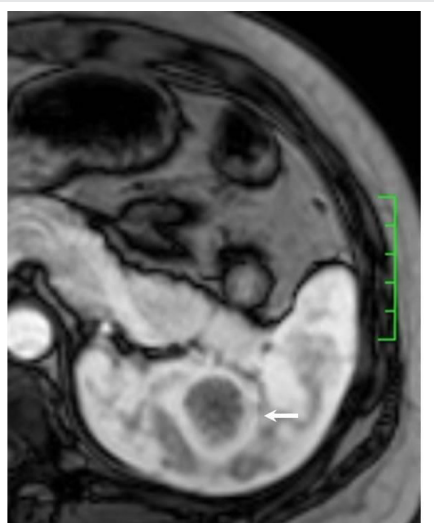

Figure 4a: 35-year-old woman with SANT. Axial contrast-enhanced T1 weighted gradient echo image in hepatic arterial phase shows avid circle enhancement (arrow)

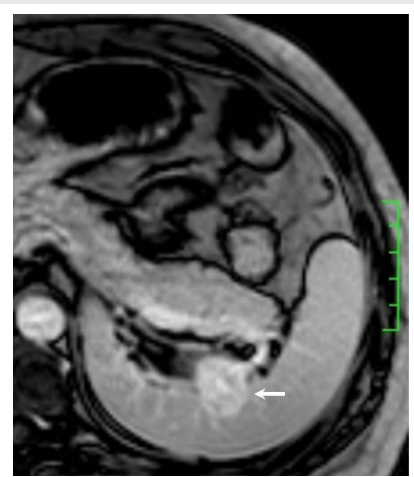

Figure 4b: 35-year-old woman with SANT. Axial contrast-enhanced T1 weighted gradient echo image in delay phase shows the persistent circle enhancement, radial lines and a central scar, which forms a spoke-like appearance(arrow). recognized earlier by other authors under different names such as splenic hamartoma, cord capillary hemangioma, and multinodular hemangioma [2]. SANTs are considered as a benign, nodular vascular proliferations of splenic red pulp due to an exaggerated stromal response rather than a true neoplastic process [3-5]. The typical macroscopic appearance of a SANT lesion was of a well-circumscribed non-encapsualted, bosselated mass with multiple dark brown nodules (hemorrhagic regions in angiomatoid nodules) interspersed with stellate whitish fibrotic stvroma.

It usually affects middle-aged adults [6] and it is commonly found incidentally on radiographic imaging, or during operation for an unrelated condition. In present series, the patients are middle-aged adults without any related symptoms and the lesions were detected incidentally at routine ultrasound of health examinations. On ultrasound, manifestations of SANTs are vary widely from heterogeneous echo-texture with hyperechogenic, hypoechogenic, or isoechogenic appearing lesions, which are unspecific $[7,8]$. CT and MRI can show some characteristic features of SANTs, which include a solitary well demarcated mass with early peripheral enhancemant with radiating lines and progressive enhancement at the delay phase, and hypointens at T2 weighted MR images [2,3,5,913]. These features reflect the underlying pathological changes of scattered angiomatoid nodules separated and surrounded by sclerotic fibrotic stroma [10]. In present series, we also observed these characteristic features with well correlation to pathological findings. Circle and spoke-like enhancement can be contributed to fibrotic stroma surrounding and separating the angiomatoid nodules. The sclerotic fibrotic stroma may also result in isodense on $\mathrm{CT}$, isointensity on $\mathrm{T} 1$ weighted images and hypointensity on $\mathrm{T} 2$ weighted images of MR imaging. The signal void spots at $\mathrm{T} 2$ weighted MR images have not been described at other literatures and may be contributed to hemosiderin deposition due to magnetic susceptibilty artifact.

As a solitary solid splenic mass, SANT should be differentiated from hemangiomas at first, which are the most common benign neoplasm of spleen [12]. At CT, it is difficult to differentiate SANT from hemoangioma due to progressive filling in enhancement, but circle enhance at hepatic arterial phase and continuing radial enhanced lines might provide some clues for SANT. MR imaging might be helpful in differential diagnosis. Unlike to SANT, hemangiomas are hyperintensity at T2 weighted MR images [3]. Angiosarcomas are rare and also of T2 high signal intensity. These two entities also lack intratumor signal void spots at $\mathrm{T} 2$ weighted images. Lymphoma is the most common malignant tumor of the spleen, but it often appears as isointense or hypointense relative to the spleen on T2weighted images. Another sign that facilitates to distinguish lymphoma from SANT is the mild to moderate homogenerous enhancement of lymphoma [13].

In conclusion, SANTs have some CT and MRI characteristics, which can facilitate to distinguish them from other splenic neoplasms. These features include T2 low signal solitary mass with scattered signal void spots, spoke-like enhancement of enhanced circle and radiate lines, obvious delay-enhancement of a progressive filling in pattern. CT 
and MRI features are well correlated to pathological findings of sclerotic fibrotic stroma and angiomatoid nodules with hemosiderin deposition. As wide spread use of cross-section imaging modalities at routine health serves, this rare entity may be come across sometimes. Familiarity with characteristic imaging findings of SANT can facilitate radiologists to make correct diagnosis and provide guideline of treatment decision for clinicians. Due to its benign features, imaging follow-up is considered a preference rather than biopsy and resection.

\section{References}

1. Martel M, Cheuk W, Lombardi L, Lifschitz-Mercer B, Chan JK, et al. (2004) Sclerosing angiomatoid nodular transformation (SANT): report of 25 cases of a distinctive benign splenic lesion. Am J Surg Pathol 28: 1268-1279. Link: https://goo.gl/j94Wj3

2. Zeeb LM, Johnson JM, Madsen MS, Keating DP (2009) Sclerosing angiomatoid nodular transformation. AJR Am J Roentgenol 192: W236-238.

3. Thipphavong S, Duigenan S, Schindera ST, Gee MS, Philips S (2014) Nonneoplastic, benign, and malignant splenic diseases: cross-sectional imaging findings and rare disease entities. AJR Am J Roentgenol 203: 315 322. Link: https://goo.gl/PKWe32

4. Diebold J, Le Tourneau A, Marmey B, Prevot S, Müller-Hermelink HK, et al (2008) Is sclerosing angiomatoid nodular transformation (SANT) of the splenic red pulp identical to inflammatory pseudotumour? Report of 16 cases. Histopathology 53: 299-310. Link: https://goo.gl/4SJHiR

5. Kim HJ, Kim KW, Yu ES, Byun JH, Lee SS, et al. (2012) Sclerosing angiomatoid nodular transformation of the spleen: clinical and radiologic characteristics. Acta Radiol 53: 701-706. Link: https://goo.gl/taUUdA
6. Pradhan D, Mohanty SK (2013) Sclerosing angiomatoid nodula transformation of the spleen. Arch Pathol Lab Med 137: 1309-1312. Link: https://goo.gl/66oK9X

7. Watanabe $M$, Shiozawa $K$, Ikehara $T$, Kanayama $M$, Kikuchi $Y$, et al (2014) A case of sclerosing angiomatoid nodular transformation of the spleen: correlations between contrast-enhanced ultrasonography and histopathologic findings. J Clin Ultrasound 42: 103-107. Link: https://goo.gl/ HrGVQL

8. Cao JY, Zhang H, Wang WP (2010) Ultrasonography of sclerosing angiomatoid nodular transformation in the spleen. World J Gastroenterol 16: 3727-3730. Link: https://goo.gl/2nCgxE

9. Yoshimura N, Saito K, Shirota N, Suzuki K, Akata S, et al. (2015) Two cases of sclerosing angiomatoid nodular transformation of the spleen with gradual growth: usefulness of diffusion-weighted imaging. Clin Imaging 39: 315-317. Link: https://goo.gl/IVRH7d

10. Thacker C, Korn R, Millstine J, Harvin H, Van Lier Ribbink JA, et al. (2010) Sclerosing angiomatoid nodular transformation of the spleen: CT, MR, PET, and (9)(9)(m)Tc-sulfur colloid SPECT CT findings with gross and histopathological correlation. Abdom Imaging 35: 683-689. Link: https://goo. $\mathrm{gl} / \mathrm{jq} 1 \mathrm{Wah}$

11. Raman SP, Singhi A, Horton KM, Hruban RH, Fishman EK (2013) Sclerosing angiomatoid nodular transformation of the spleen (SANT): multimodality imaging appearance of five cases with radiology-pathology correlation. Abdom Imaging 38: 827-834. Link: https://goo.gl/C4Mc8M

12. Warshauer DM, Hall HL (2006) Solitary splenic lesions. Semin Ultrasound CT MR 27: 370-388. Link: https://goo.gl/HOWRxJ

13. Li M, Zhang L, Wu N, Huang W, Lv N (2013) Imaging findings of primary splenic lymphoma: a review of 17 cases in which diagnosis was made at splenectomy. PLoS One 8: e80264. Link: Link: https://goo.gl/7hfCiE 